\title{
THE 11 MICRON EMISSIONS OF CARBON STARS
}

J. H. GOEBEL, P. CHEESEMAN, and F. GERBAULT 
The Astrophysical Journal, 449:246-257, 1995 August 10

(C) 1995. The American Astronomical Society. All rights reserved. Printed in U.S.A

\title{
THE 11 MICRON EMISSIONS OF CARBON STARS
}

\author{
J. H. Goebel, ${ }^{1}$ P. Cheeseman, ${ }^{2}$ and F. Gerbault ${ }^{3}$ \\ Received 1994 October 31 ; accepted 1995 February 15
}

\begin{abstract}
A new classification scheme of the IRAS LRS carbon stars is presented. It comprises the separation of 718 probable carbon stars into 12 distinct self-similar spectral groupings. Continuum temperatures are assigned and range from 470 to $5000 \mathrm{~K}$. Three distinct dust species are identifiable: $\mathrm{SiC}, \alpha: \mathrm{C}-\mathbf{H}$, and $\mathbf{M g S}$. In addition to the narrow $11+\mu \mathrm{m}$ emission feature that is commonly attributed to $\mathrm{SiC}$, a broad $11+\mu \mathrm{m}$ emission feature, that is correlated with the 8.5 and $7.7 \mu \mathrm{m}$ features, is found and attributed to $\alpha: \mathrm{C}-\mathrm{H}$. SiC and $\alpha: \mathrm{C}-\mathrm{H}$ band strengths are found to correlate with the temperature progression among the Classes. We find a spectral sequence of Classes that reflects the carbon star evolutionary sequence of spectral types, or alternatively developmental sequences of grain condensation in carbon-rich circumstellar shells. If decreasing temperature corresponds to increasing evolution, then decreasing temperature corresponds to increasing $\mathrm{C} / \mathrm{O}$ resulting in increasing amounts of carbon rich dust, namely $\alpha: C-H$. If decreasing the temperature corresponds to a grain condensation sequence, then heterogeneous, or induced nucleation scenarios are supported. $\mathrm{SiC}$ grains precede $\alpha: \mathrm{C}-\mathrm{H}$ and form the nuclei for the condensation of the latter material. At still lower temperatures, MgS appears to be quite prevalent. No $11.3 \mu \mathrm{m}$ PAH features are identified in any of the 718 carbon stars. However, one of the coldest objects, IRAS 15048-5702, and a few others, displays an $11.9 \mu \mathrm{m}$ emission feature characteristic of laboratory samples of coronene. That feature corresponds to the $\mathrm{C}-\mathrm{H}$ out of plane deformation mode of aromatic hydrocarbon. This band indicates the presence of unsaturated, $s p^{3}$, hydrocarbon bonds that may subsequently evolve into saturated bonds, $s p^{2}$, if, and when, the star enters the planetary nebulae phase of stellar evolution. The effusion of hydrogen from the hydrocarbon grain results in the evolution in wavelength of this $11.9 \mu \mathrm{m}$ emission feature to the $11.3 \mu \mathrm{m}$ feature.
\end{abstract}

Subject headings: circumstellar matter - infrared: stars - molecular processes - stars: carbon stars: fundamental parameters

\section{INTRODUCTION}

Historically, silicon carbide ( $\mathrm{SiC}$ ) was the first dust species identified from its infrared spectroscopic features in carbon stars (Treffers \& Cohen 1974). The $11 \mu \mathrm{m}$ band profile has been observed from ground based observatories in a large number of carbon stars by Forrest, Gillett, \& Stein (1975) and by Cohen (1979). Whereas Forrest et al. (1975) found a fairly uniform band profile among the visually bright carbon stars, Cohen (1980) observed subtle differences in cold dust enshrouded carbon stars, those he termed extreme carbon stars. In the bright visible carbon star Y CVn, Goebel et al. (1980) observed a somewhat different $\mathrm{SiC}$ band profile than normally found in the other visible carbon stars that was comparable to that expected from the crystalline forms of $\mathrm{SiC}$ produced in the laboratory by Dorschner, Friedemann, \& Gürtler $(1977 \mathrm{a}, \mathrm{b})$. Until the IRAS Atlas became available, there were available for study relatively few spectra of the $11 \mu \mathrm{m} \mathrm{SiC}$ band. With the advent of the LRS, there are hundreds of spectra with good signal-to-noise ratio and spectral coverage in the important $15 \mu \mathrm{m}$ region that is blocked from ground and airborned observation. The characteristics of the band profiles have been the subject for discussion by Papoular (1988), LittleMarenin et al. (1987), and Skinner \& Whitmore (1988a, b).

\footnotetext{
' Sensors and Instrumentation Branch, Space Projects Division, NASA Ames Research Center, MS 244-10, Moffett Field, CA 94035

${ }_{2}$ Research Institute for Advanced Computer Science, NASA Ames Research Center, MS 269-2, Moffett Field, CA 94035.

${ }^{3}$ Sterling Federal Systems, NASA Ames Research Center, MS 244-10, Moffett Field, CA 94035.
}

$\mathrm{SiC}$ has been incorporated into models and is currently studied in the laboratory as a carbon star circumstellar material. SiC particles have been studied extensively in the laboratory by Dorschner et al. (1977a, b), Friedemann et al. (1981), Borghesi et al. (1983), Borghesi et al. (1985), Pégourié (1988), and Lewis, Amari, \& Anders (1990) with the astrophysical application in mind. Radiative transfer models for stellar carbon rich circumstellar shells concentrated mainly on the carbon dust component because of the significant absorption coefficient it displays at nearly all infrared wavelengths, and treated $\mathrm{SiC}$ as an impurity by mass and a minor contributor of opacity, and therefore, to the balance of energy in the radiation transfer equation (Martin \& Rogers 1987). In most modeling of carbon stars, the $\mathrm{SiC}$ feature and component by mass is relegated to insignificance (e.g., Rowan-Robinson \& Harris 1983; Le Bertre 1988). To the contrary, the models of Chan \& Kwok (1990) presume only an empirical SiC opacity without carbon dust that can model the radiative transfer successfully in optically thin and thick shells. The peak in the spectral energy distribution of some planetary nebulae occurs in the 8 to $14 \mu \mathrm{m}$ region, so that $\mathrm{SiC}$ emission feature can represent a significant fraction of the total radiation in the spectrum. See Hoare (1990) for a recent application of dust models that examine the dust-to-gas ratio in carbon-rich planetary nebulae.

The observational studies of the $\mathrm{SiC}$ component in meteoritic specimens by Tang \& Anders (1988), Zinner, Tang, \& Anders (1989), and Amari et al. (1992) indicate the presence of $\mathrm{SiC}$ outside the confines of a carbon-rich circumstellar environment and furthermore are of importance to our understanding of the early solar nebula. The larger meteoritic SiC 

grains contain rare gas isotropes consistent with those that are expected to be present in the asymtotic giant branch (AGB) He-burning shell and the envelope (Lewis et al. 1990; Gallino et al. 1994; Hoppe et al. 1994). The smaller meteoritic SiC particles also appear to be possible carbon star ejecta with s-process enhancements of isotopes (Brown \& Clayton 1992). Another $\mathrm{SiC}$ component with unusual isotopic composition, grains " $X$," may be formed in the explosive ejecta of supernovae (Amari et al. 1992; Hoppe et al. 1994). All the SiC grains examined by Hoppe et al. (1994) are cubic $(\beta-\mathrm{SiC})$ based on $\mathrm{X}$-ray diffraction, yet amazingly many different grain forms are displayed.

Historically, the question of the presence of SiC in the interstellar medium has been of considerable interest, and its puzzling absence in the interstellar extinction curve is the subject of a recent discussion by Whittet, Duley, \& Martin (1990). They set an upper limit on the mass fraction of $\mathrm{SiC}$ in interstellar dust of at most a few percent, arguing that it is oxidized in the interstellar diffuse cloud environment on a timescale of $\sim 5 \times 10^{7}$ years.

It is the purpose of this work to demonstrate that there is a progression of $11 \mu \mathrm{m}$ emission features in carbon rich stars that indicate the presence of material components other than $\mathrm{SiC}$. Spectral classes are defined by the use of automatic classification procedures and can be understood in terms of multiple grain types. It is possible to find reasonable spectral identifications for the emission features that are consistent with physical conditions in and evolutionary history of carbon stars.

\section{A NEW CLASSIFICATION OF LRS CARBON STARS}

Previously, we used the artificial intelligence classification technique AutoClass II developed by Cheeseman et al. (1988) to automatically classify all the spectra in the Atlas of LowResolution IRAS Spectra (1986, hereafter the IRAS LRS Atlas). This is in contrast to the human expert technique used by the authors of the IRAS Atlas that was based on the obvious concept of spectral similarity. The study upon which we report here is an extension of the previous Cheeseman et al. (1988) work and focuses only on the groups identified as carbon star Classes. It includes 718 objects which we will call probable carbon stars. The result of this effort is summarized in Table 1 ; we find a total of 12 separate classes of carbon stars in the LRS. AutoClass II has produced 12 carbon star Classes from a sample of 718 . A total of 10 or 11 classes are expected by application of Sturges's rule for classifying a series of $N$ items (Newman, Haynes, \& Terzian 1994). AutoClass II appears to be finding statistically significant differences in the population, not arbitrary groupings.

The objects are drawn from the MetaClasses $\alpha 1-\alpha 6, \delta 7, \lambda 20$, and $\lambda 30: 1$ and $\lambda 30: 5$ of Cheeseman et al. (1988). Also included were all those carbon stars identified in the catalog association field (catalog 17; Stephenson 1973) of the IRAS LRS Atlas distribution tape even if not included in the carbon star MetaClasses, e.g., BM Gem, which is in a silicate emission Class (11). It would be silly to intentionally exclude visibly identified carbon stars, for that is totally inconsistent with the Bayesian principles of incorporating all prior probabilities. In retrospect, we have missed a small number of stars which did not satisfy these criteria like $08445-3932=$ HD 75021, since we assumed all visibly bright identified carbon stars were included in the $\mathrm{CCCS}=$ Catalog 17 . Hence, we are not claiming absolute completeness for the list of carbon stars presented here. Not included were MetaClasses $\gamma$ and $\zeta$, among which are found some protoplanetaries and many planetaries, since we did not wish to expand the working definition of carbon stars to include both those groupings. Moreover, their spectra are sufficiently similar to those of silicate absorption shells so that the LRS instruments give little distinction between the two groups. The reference work by Cheeseman et al. (1989) provides a separate listing of planetary nebulae and protoplanetary objects from the LRS that are primarily resident in the $\gamma$ MetaClass.

Considering only the MetaClass members, we find 161 out of 176 stars found in the list of Little-Marenin et al. (1987) of new carbon stars and 57 out of 65 of the carbon stars listed by her as well known. When catalog 17 association is included, all their previously determined carbon stars in the LRS Atlas are recovered. In addition, there are objects in our list that are new associations with carbon stars, determined solely upon the basis of the AutoClass search reported here. Most of these 225 probable carbon stars are of low signal-to-noise ratio. Their validity needs to be checked at near infrared wavelengths by spectroscopic means. We believe that the vast majority of carbon stars have been included in this study, and that the few

TABLE 1

Summary of AutoClass II Carbon Star Characteristics

\begin{tabular}{|c|c|c|c|c|c|c|c|c|c|}
\hline \multirow[b]{2}{*}{ Class } & \multirow[b]{2}{*}{ Population } & \multirow[b]{2}{*}{$\begin{array}{c}T_{c} \\
(\mathrm{~K})\end{array}$} & \multirow[b]{2}{*}{ Dust/Gas SPECIES } & \multirow[b]{2}{*}{$\begin{array}{l}\text { LRS Classes } \\
\text { (DOMINANT) }\end{array}$} & \multirow{2}{*}{$\begin{array}{l}\text { GaLACtIC } \\
\text { LATITUDE } \\
\text { DisPERSION }\end{array}$} & \multirow{2}{*}{$\begin{array}{l}\text { MAG } \\
{[12]}\end{array}$} & \multicolumn{3}{|c|}{ FIR COLORS } \\
\hline & & & & & & & {$[12]-[25]$} & {$[25]-[60]$} & {$[60]-[100]$} \\
\hline $0 \ldots \ldots$ & 109 & 800 & $\mathrm{SiC}+\alpha: \mathrm{C}-\mathrm{H}$ & $4 n$ & $15^{\circ} 234$ & -1.053 & 0.462 & 0.229 & 0.253 \\
\hline $1 \ldots \ldots \ldots$ & 103 & 600 & $\alpha: \mathrm{C}-\mathrm{H}+\mathrm{SiC}+\mathrm{MgS}$ & $4 n$ & 6.344 & -1.119 & 0.867 & 0.256 & 0.310 \\
\hline $2 \ldots \ldots \ldots$ & 74 & 5000 & Unknown & $0 \mathrm{n}, 1 \mathrm{n}$ & 14.288 & 1.070 & 0.528 & 0.328 & $2.511^{\circ}$ \\
\hline $3 \ldots \ldots \ldots$ & 74 & 2000 & Gas + dust & $4 n, 1 n$ & 20.679 & -0.428 & 0.262 & 0.531 & 0.750 \\
\hline $4 \ldots \ldots \ldots$ & 73 & 2000 & $\mathrm{SiC}$ & $4 n, 1 n$ & 9.365 & 0.324 & 0.492 & 0.342 & 1.113 \\
\hline $5 \ldots \ldots \ldots \ldots$ & 60 & 470 & $\alpha: C-H+M g S$ & $4 n, 1 n$ & 7.739 & -1.016 & 1.222 & 0.395 & -0.175 \\
\hline $6 \ldots \ldots \ldots$ & 58 & 5000 & Gas + dust & $1 \mathrm{n}, 0 \mathrm{n}, 4 \mathrm{n}$ & 11.638 & 0.764 & 0.344 & 0.456 & 1.735 \\
\hline $7 \ldots \ldots \ldots$ & 46 & 5000 & Gas + dust & On, $\ln , 4 n$ & 7.929 & 1.175 & 0.419 & 0.404 & $2.140^{b}$ \\
\hline $8 \ldots \ldots \ldots$ & 39 & 900 & $\mathrm{SiC}+\alpha: \mathrm{C}-\mathbf{H}$ & $\ln , 4 n$ & 4.256 & 0.790 & 0.653 & 0.374 & \\
\hline $9 \ldots \ldots \ldots$ & 39 & 600 & $\alpha: C-H$ & $\ln , 4 \mathrm{n}$ & 8.547 & 0.554 & 0.570 & 0.533 & $0.960^{\mathrm{c}}$ \\
\hline $10 \ldots \ldots \ldots$ & 29 & & Unknown & On, $2 \mathrm{n}$ & 12.071 & 1.373 & 0.731 & 0.748 & $1.861^{\mathrm{d}}$ \\
\hline $11 \ldots \ldots \ldots$ & 6 & 600 & Gas/silicates & $2 n, \ln , 4 n$ & 8.119 & 0.318 & 0.839 & 0.022 & 0.181 \\
\hline
\end{tabular}

- Only two stars with reliable [60]-[100]. Must be considered an upper limit.

- Only three stars with reliable [60]-[100]. Must be considered an upper limit.

- Only four stars with reliable [60]-[100]. Must be considered an upper limit.

- Only one star with reliable [60]-[100]. Must be considered an upper limit. 
that have been overlooked will not greatly affect the conclusions based upon looking at the groups, rather than individuals.

The parameters of the 12 carbon star Classes are summarized in Table 1. "Class" is the AutoClass Class number; "Population" is the Class membership count; $T_{c}$ is the estimated Class continuum temperature; " Dust/Gas Species" lists the dust species assigned here; "LRS Classes" lists the dominant LRS Classes present in an AutoClass Class; "Galactic Latitude Dispersion " is the standard deviation in the distribution of galactic latitude of members of the Class; " $\operatorname{mag}$ [12]" is the IRAS band 1 flux magnitude average of the Class as defined in Walker \& Cohen (1988) and Cheeseman et al. (1989); and "FIR Colors" are the IRAS band 2, 3, and 4 color differences of the Class average in magnitudes as defined in Cheeseman et al. (1989).

The temperatures quoted in Table 1 are derived by comparison of the flux curves with single temperature blackbody functions, as distinguished from a more complex radiative transfer calculation involving dust opacities. The fitting criteria are unique to each Class taking into account the different characteristic spectral features of each Class. Temperature assignment represents post-AutoClass analysis. Of course noisy Classes have less reliable temperature estimates of the continuum, $T_{c}$, than quiet Classes. The absolute temperature scale is somewhat suspect because of the photometric calibration procedures in the original IRAS LRS Atlas which used $\alpha$ Tau as the primary standard and assigned to it a temperature of $10,000 \mathrm{~K}$, as the accepted temperature of $\alpha$ Tau is more like $4500 \mathrm{~K}$. We have not attempted to correct for the temperature calibration error present in the data from the Atlas in order to maintain consistency with the data set presented there. Due to the presence of $\mathrm{SiO}$ absorption in the $8 \mu \mathrm{m}$ region, the red end of each and every spectrum is about $10 \%$ higher in flux than it should be. Approximate adjustment can be made by using the ratio of 10,000 and $4500 \mathrm{~K}$ blackbodies in the appropriate fashion. Recalibrations are discussed by Wooden et al. (1993). The more exact correction can be made using the recalibration table of Cohen et al. (1992). Our choice to maintain consistency with the LRS atlas is not without penalty. We may have missed identifying those stars that have intrinsic $\mathrm{SiO}$ absorption at 8 $\mu \mathrm{m}$. SiO is predicted to be abundant in certain elemental abundance circumstances in carbon stars (Tsuji 1964, 1973), and has been identified at $4 \mu \mathrm{m}$ in the spectrum of TX Psc by Bregman, Goebel, \& Strecker (1978).

In Figure 1, we compare the spectra of Classes 0, 1, 3, 4, and 5 with the laboratory spectra of $\mathrm{SiC}$ and $\alpha: \mathrm{C}-\mathrm{H}$. The Classes have been arranged from top to bottom in order of increasing $8.5 \mu \mathrm{m}$ emission strength and decreasing $11+\mu \mathrm{m}$ emission strength. This sequence corresponds to a continuum temperature, $T_{c}$, sequence decreasing with increasing $8.5 \mu \mathrm{m}$ emission excess, with the exception of Class 3 . Analysis of Class 3 shows that it is complicated by the presence of molecular absorption bands (Goebel et al. 1988), so we can understand its deviation from the $T_{c}$ sequence on this basis. We consider there to be a good $T_{c}$ sequence for the Classes which are apparently dominated by dust, i.e., $0,1,4$, and 5 .

From the comparison of Class spectra with material emittances that is displayed in Figures 2 and 3, we can conclude that $\mathrm{SiC}$ predominates in the higher temperature spectra, while $\alpha: \mathrm{C}-\mathrm{H}$ dominates at the lower temperatures. At intermediate temperatures, $500 \mathrm{~K}<T_{c}<2000 \mathrm{~K}$, and excluding Class 3 for the stated reasons, there appears to be a mixture of the fea-

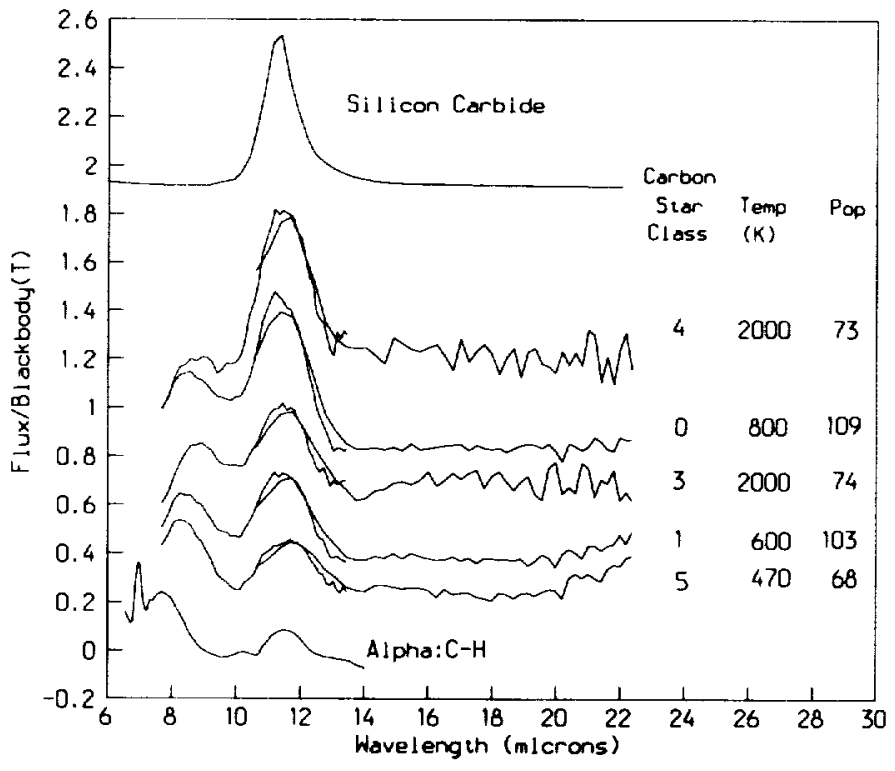

Fig. 1.-The average spectra of Classes $0,1,2,3,4$, and 5 , the most populous carbon star Classes, normalized to their respective continuum temperatures. Also displayed are the arbitrarily normalized absorption coefficients of $\beta$ - $\mathrm{SiC}$ and $\alpha: \mathrm{C}-\mathrm{H}$ for comparison purposes.

tures. The mixture causes the peak wavelength and width of the $11+\mu \mathrm{m}$ feature to shift from Class to Class. We conclude that the shift cannot be due to temperature effects on the $\mathrm{SiC}$ emission band, because the predicted broadening of the band profile increases with increasing temperature. This is in opposition to the observed trend. In Table 1, we have listed the dust components that appear to be correlated with each of the 12 Classes. Some of the Classes, like 8 and 9 , are low $\mathrm{S} / \mathrm{N}$ versions of Classes $0,1,4$, and 5 .

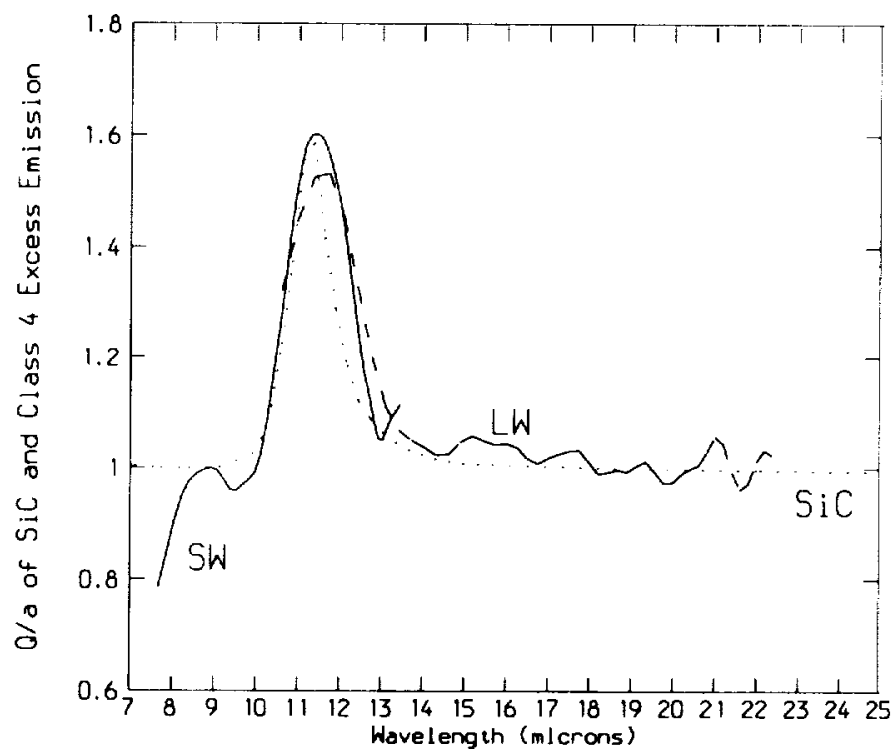

Fig. 2.-The average normalized emission excess spectrum of carbon star Class 4 compared directly with the absorption coefficient of $\beta-\mathrm{SiC}$ normalized to the same $11+\mu \mathrm{m}$ peak height as Class 4 . SW and LW refer to the short and long wavelength data sets of the IRAS LRS spectrometer. 


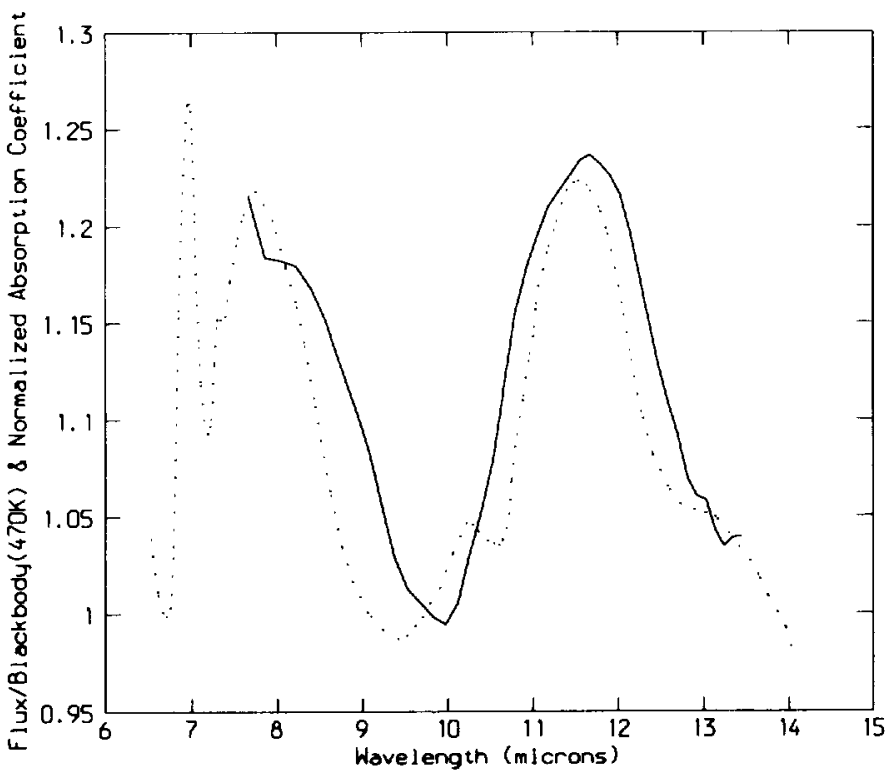

Fig. 3.-The average normalized emission excess spectrum of carbon star Class 5 (solid and dashed curves) directly compared with the absorption coefficient of $\alpha: \mathrm{C}-\mathrm{H}$ (dotted curve) normalized to the same $11+\mu \mathrm{m}$ peak height as Class 5.

\subsection{Other LRS Classifications of Carbon Stars}

Little-Marenin et al. (1987) have classified 176 LRS spectra as carbon stars using the $11.2 \mu \mathrm{m} \mathrm{SiC} \mathrm{emission} \mathrm{as} \mathrm{the} \mathrm{selection}$ feature. They compared an appropriate blackbody function to each spectrum and examined the remaining excesses for the characteristic $11.2 \mu \mathrm{m}$ emission profile. Our examination has recovered all of the bona fide entries in their Tables 1 and 2 of known and previously unknown carbon stars. Outside the scope of the present discussion are the puzzling few carbon stars with silicate-like emission features. Both Little-Marenin (1986) and Willems \& de Jong (1986a, b) have discussed the discovery and implications of these spectra. AutoClass II also recognizes these spectra and places them in a separate Class 11 of Table 1 .

Willems (1987) considered the 304 carbon star spectra listed in the LRS Atlas. He provided an $\mathrm{SiC}$ index for those with an emission feature peaking between 11.2 and $11.6 \mu \mathrm{m}$. He recognized spectra with featureless dust emission outside the $\mathrm{SiC}$ feature attributed to amorphous carbon dust. Other spectra exhibited absorption bands due to molecular species, primarily $\mathrm{HCN}$ and $\mathrm{C}_{2} \mathrm{H}_{2}$. Those with near-infrared color temperatures, $T_{\mathrm{IR}}>2000 \mathrm{~K}$, have optically thin dust emission shells in the continuum and frequently display the $\mathrm{SiC}$ feature as well as molecular absorptions; while those with $T_{\mathrm{IR}}<2000 \mathrm{~K}$ have optically thick dust emission shells in the continuum and exhibit emission from dust, either from $\mathrm{SiC}$ or from amorphous carbon, or both, along with molecular features. Since the maximum $\mathrm{SiC}$ index was 0.24 , the interpretation was given that only a limited fraction of the carbon was condensed in the form of $\mathrm{SiC}$, of order $1 \%-2 \%$ of the dust particles. The variation in shape of the $\mathrm{SiC}$ feature was considered to be consistent with the well-known sensitivity of the feature to particle shape, as was discussed by Treffers \& Cohen (1974). The limit of the classification effort to optically thin and thick continuum shells, largely left unaddressed the issues to be discussed here, namely the progression of emission features within the LRS sample.
Popular (1988) has classified the infrared spectra of carbon stars in the LRS following a different procedure than ours. He observed three different types of $11.3 \mu \mathrm{m}$ features differing in width and by the occurrence of secondary features at $\sim 8.6$, $\sim 11.7$, and $12.8 \mu \mathrm{m}$. The different $\mathrm{SiC}$ features could be matched by pure or polluted $\alpha$-SiC from the laboratory studies of Bohrgesi et al. (1985). The classification procedure was that of Baron et al. (1987). First an underlying continuum was estimated based on a power law connecting two spectral points in the short-wavelength portion of the spectrum as chosen by the observer. The resulting spectral excess and feature shape were defined mathematically. Then the individual spectra were ranked according to excess and shape of the $\mathrm{SiC}$ feature, nominally at $11.3 \mu \mathrm{m}$. The resultant spectra displayed a wide range of behaviors in the long wavelength region that were primarily due to the estimation of the continuum by a power law rather than a blackbody. However, in the shortwavelength region, the feature shapes have much in common with the feature shapes in the AutoClass II Classes of the present work. For example, his subset $\mathrm{SiC}(\mathrm{a})$ is similar to our Class 0 and subset $\mathrm{SiC}(\mathrm{b})$ is similar to Class 4 . There exist significant differences as well. Subset $\mathrm{SiC}$ (c) has no counterpart in our study. The closest we come to such a spectral shape is Class 11, the Class which contains the few stars with silicate type emission. While we also use an estimate of the continuum and remove it to study the spectral features, we chose blackbody functions because of the significant spectral curvature in the 8 to $24 \mu \mathrm{m}$ wavelength range of the functions in the lowest range of temperatures appropriate to these stars.

The classification by AutoClass II was done in flux versus wavelength space, not blackbody normalized excess versus wavelength space, so that the temperature of the objects would be a consideration in the procedure. Papoular's procedure removes the influence of temperature prior to classification. Therefore any influence of temperature on the spectral features is lost. Additionally, the starting set of objects in the two studies are significantly different. Our set was selected primarily based upon the previous AutoClass II examination of the entire LRS Atlas, whereas Papolar's set was selected by the eye of an expert individual, rather than a mathematically rigorous algorithm. For these reasons, our results are significantly different.

Chan \& Kwok $(1988,1990)$ studied 356 carbon stars from the General Catalog of Cool Carbon Stars (Stephenson 1973) by examining the IRAS far-infrared photometry and the LRS spectrophotometry of the 538 objects listed in LRS Class $4 \mathrm{n}$. The sample was paired down to 356 for various reasons. The extent of their classification study was to redefine the spectral index with the result that none of the $\mathrm{SiC}$ emitting spectra had indicies exceeded their index 45 . They studied the differences displayed in the characteristics of the $\mathrm{SiC}$ emission feature in the visually bright and infrared bright carbon stars. The differences in the SiC spectra of the two groups were interpreted to express an evolution from $\alpha-\operatorname{SiC}$ to $\beta$-SiC parallel to the evolution from visually bright to infrared bright carbon star. Our approach to classification and spectral analysis differ significantly from their work, and we come to distinctly different conclusions regarding the nature of the dust emitting materials.

Volk \& Cohen (1989) examined the LRS database from which the LRS Atlas was extracted, and found 365 highquality spectra that for one reason or another were not included in the IRAS LRS Atlas. Of the reported sources, 28 
were determined to be $\mathrm{SiC}$ emitters and some dozen others possibly carbon stars. The large number of potentially carbonrich planetary nebulae that they found are not considered here. Of the nearly $\mathbf{4 0}$ carbon stars outside the LRS Atlas, some are well-known visibly bright carbon stars, e.g., TX Psc, V CrB, $\mathrm{S}$ Cep, and V Hya. They have not been included in our analysis of the Atlas, because we examined the IRAS distribution product, or tapes, not the hours-confirmed-sources, or raw data distribution on CD-ROMs. Several of these extra sources are included in the study by Skinner \& Whitmore (1988a, b) of the $\mathrm{SiC}$ feature and its relation to mass-loss rates.

\section{DISCUSSION}

We divide the discussion into three sections. The first section discusses the similarities shown by the LRS spectra with laboratory spectra of relevant materials and the dissimilarities of other LRS emission features. The second section discusses the evolutionary theories proposed here and elsewhere. The third section deals with the implications of the present interpretation for dust condensation theories.

\subsection{Laboratory and Astronomical Spectra}

In Figure 2, we present a direct comparison of the $\mathrm{SiC}$ emission with that of Class 4 . The $\alpha-\mathrm{SiC}$ emission is from the work by Pégourié (1988). The $300 \mathrm{~K}$ temperature of the $\mathrm{SiC}$ band produces an understandably narrower emission profile than the $2000 \mathrm{~K}$ continuum of Class 4 might imply. While it is not obvious what temperature to assign to the emission of Class 4's $\mathrm{SiC}$ profile, it appears to be warmer than $300 \mathrm{~K}$, and, therefore, broader than the laboratory sample, specifically on the long wavelength side, rather than the short wavelength side of the feature. The sharp cutoff on the short wavelength side of the feature disallows a strong temperature influence on that part of the feature and accounts for the similarity of that profile. The slower cutoff on the long wavelength side allows the temperature to influence the feature width. It would be interesting to model the shape and temperature of the $\mathrm{SiC}$ grains to determine the effect of these parameters on the profile of the band.

The $\alpha: \mathrm{C}-\mathrm{H}$ absorption spectrum in Figures 1 and 3 is from Dischler et al. (1983a, b). It compares most favorably with the spectrum of Class 5, the Class with the lowest continuum temperature assignment, $470 \mathrm{~K}$. The direct comparison of Class 5 with $\alpha: \mathbf{C}-\mathrm{H}$ is presented in Figure 3. The reported laboratory absorption shown in Figure 1 displays stronger absorption at $7.8 \mu \mathrm{m}$ than at $11+\mu \mathrm{m}$. Normalizing the laboratory bandstrengths to a $300 \mathrm{~K}$ excitation temperature gives nearly equal bandstrengths. In Figure 3, the temperature normalized bands of the laboratory material and the temperature normalized bands of the stellar spectrum compare very well in central wavelength and symmetry. The central wavelength of $\alpha: C-H$ is $11.55 \mu \mathrm{m}$, and that of the feature in Class 5 is $11.67 \mu \mathrm{m}$. Minor differences are apparent in the width of the feature at $11+\mu \mathrm{m}$ and the shape of the base of the feature. In Figure 1, the sharp turndown in LRS spectra short of $8 \mu \mathrm{m}$, that is an artifact of the LRS instrument, has been corrected using the recalibration of Cohen, Walker, \& Witteborn (1992). Since the correction to spectra is greatest near $8 \mu \mathrm{m}$, and that region involves the $7.8 \mu \mathrm{m}$ band of $\alpha: \mathrm{C}-\mathrm{H}$, the corrected spectrum of Class 5 is used in Figure 3.

As the Class temperature increases in Figure 1, so does the apparent strength of the $11+\mu \mathrm{m}$ emission relative to that at 8 $\mu \mathrm{m}$, with the exception of Class 3 , about which more will be said later. The central wavelength of this feature also moves progressively to shorter wavelengths with increasing temperature. This can be interpreted in terms of a two-component model that mixes $\mathrm{SiC}$ and $\alpha: \mathrm{C}-\mathrm{H}$, and favors $\mathrm{SiC}$ at higher temperatures and $\alpha: \mathrm{C}-\mathrm{H}$ at lower temperatures. The interpretation here is distinctly different from that arrived at by Papoular (1988).

The apparent similarity of the two $11+\mu \mathrm{m}$ peaks for the two materials $\mathrm{SiC}$ and $\alpha: \mathrm{C}-\mathrm{H}$ leads to a natural explanation for the narrow and wide $11+\mu \mathrm{m}$ features observed by Cohen (1979) in carbon-rich dust shells. The narrow feature is attributable to $\mathrm{SiC}$ and the broad feature corresponds to $\alpha: C-H$. SiC's emission feature cuts off at a shorter wavelength on the long wavelength side of its emission feature than does that of $\alpha: C-H$, see Figures 2 and 3. The alternative explanations for differences in the $11+\mu \mathrm{m}$ feature involve shape and size differences in $\mathrm{SiC}$ grains that influence the radiation cross section, or radiation induced damage effects on the crystalline structure. Because there are so many possible configurations of grain shape, size, and damage parameters, these explanations should produce a continuum of classes, whereas, in our analysis we observe a few distinct classes.

The four Classes $0,1,4$, and 5 represent $49 \%$ of all the AutoClass carbon stars, so we have an explanation for the characteristic dust emission profiles of a major fraction of all carbon stars in terms of two species. Classes 8 and 9, with an additional $11 \%$ of the population, are of similar characteristics but with lower signal to noise ratio. Hence, the majority of carbon stars, at least $60 \%$, can be described by a duality of dust species, $\alpha-\mathrm{SiC}$ and $\alpha: \mathrm{C}-\mathrm{H}$.

A third type of $11.3 \mu \mathrm{m}$ feature occurs only in the planetary nebulae and $\mathrm{H}$ II regions where there is significant ultraviolet radiation present. It is one of the well known and long studied UIR emission features. The $11.3 \mu \mathrm{m}$ band is narrower than the $\mathrm{SiC}$ or $\alpha: \mathrm{C}-\mathrm{H}$ emission bands. It has been attributed to polycyclic aromatic hydrocarbons (PAHs) (Léger \& Puget 1984; Allamandola, Tielens, \& Barker 1985). The LRS Class of these objects is $8 \mathrm{n}$. Only two $8 \mathrm{n}$ objects, both members of Class 10 , are found in the AutoClass carbon stars. The spectra of these two objects resemble hot continua with cosmic ray spikes rather than true $11.3 \mu \mathrm{m}$ features. They may not be bona fide carbon stars. The lack of $8 \mathrm{n}$ Class objects in our sample arises from the preselection filtering of AutoClass MetaClasses done in the work of Cheeseman et al. (1989) that forms the basis of the present AutoClass carbon star set. The $\alpha$ MetaClass is predominantly populated by known carbon stars, i.e., those with catalog 17 (the CCCS) associations. These objects tend to have warm temperatures greater than $400 \mathrm{~K}$ and not the lower temperatures associated with the infrared spectra of planetaries. Neither selection of objects specifically precluded LRS 8n membership, so apparently, the narrow $11.3 \mu \mathrm{m}$ feature is not associated with the circumstellar shells of carbon stars warmer than $400 \mathrm{~K}$.

It is noteworthy that hydrogen deficient $\mathrm{R} \mathrm{CrB}$ type stars, which have enough ultraviolet radiation to be observable by $I U E$, and are present in the AutoClass carbon stars, do not display the narrow $11.3 \mu \mathrm{m}$ or the $8.5 \mu \mathrm{m}$ emissions that are indicative of bounded hydrogen (Forrest 1974; Treffers et al. 1976; Goebel et al. 1981; IRAS LRS Atlas). Therefore, the $\mathrm{R} \mathrm{CrB}$ form of carbon dust must be more graphitic (Hartmann \& Apruzese 1976) and deficient in hydrogen-and therefore, featureless in the infrared-than the hydrogenated tetrahedrally coordinated, or diamond like, $\alpha: \mathrm{C}-\mathrm{H}$ found in the majority of carbon stars that display the 8.5 and $11+\mu \mathrm{m}$ 
emission features. The graphitic form may be crystalline, or amorphous, or glassy, and must certainly be hydrogen poor. Without attached hydrogen atoms, there is no possibility of an $11.3 \mu \mathrm{m}$ emission, even with ultraviolet excitation. A lack of hydrogen in $\mathrm{R} \mathrm{CrB}$ and other related hydrogen deficient stars is well known from other studies (see Warner 1967; Fadeyev 1983; Walker 1986; Buss et al. 1993).

The narrow $11.3 \mu \mathrm{m}$ feature is currently associated with PAHs (Léger \& Puget 1984; Allamandola et al. 1985). Our association of the broad $11+\mu \mathrm{m}$ band with $\alpha: \mathrm{C}-\mathrm{H}$ does not preclude the possibility that PAHs coexist with the dust, nor that a spectrum of particle sizes ranging from molecules to dust grains exists in the circumstellar shell. In the work of Goebel (1987) a mixed population of PAHs, HACs, and graphitic carbon were proposed to explain the infrared spectrum of planetary nebula NGC 7027. Subsequent studies by Bregman et al. (1989), Witteborn et al. (1989), and Buss et al. (1993) have added further support to the idea of coexisting populations. In NGC 7027 the narrow features were attributed to PAHs and excited by ultraviolet to a high temperature like $1000 \mathrm{~K}$, while the broad underlying features (sometimes referred to as pedestals) were attributed to $\alpha: \mathrm{C}-\mathrm{H}$ and emitted at a moderate temperature like $300 \mathrm{~K}$. The graphite component accounted for the far infrared continuum, which is featureless and radiates at $90 \mathrm{~K}$ with $\lambda^{-2}$ emissivity (Forrest, McCarthy, \& Houck 1981). The ultraviolet radiation necessary to excite the narrow features like that at $11.3 \mu \mathrm{m}$ is not present in the red giant phase of carbon stars. Even when there is a hot binary star present, as is thought to be the case for UV Aur, no narrow features are observed. None of the binary carbon stars in the list of Richer (1970) are in LRS Class 8n. Apparently, binary carbon stars do not have hot enough, or intense enough ultraviolet radiation fields to excite the features. The classical carbon stars do not display the narrow emission features. If the PAHs are abundant in these objects, they have to emit copiously at $11.3 \mu \mathrm{m}$, just like any other species must emit that has permitted vibrational bands in the infrared. Since the PAHs are essentially molecules, we can count the number of photons emitted by them at $11.3 \mu \mathrm{m}$, and measure their population. The result is essentially nil. This observation certainly limits the abundance of PAH's in the atmospheres or circumstellar shells of classical carbon stars.

On the other hand, one of the coldest objects of the present study (see Table 2), 15084-5702, Class 5, which is unassociated with any major catalog, shows the $11.85 \mu \mathrm{m}$ narrowband characteristic of some laboratory PAH samples such as coronene (see Fig. 4). The $11.85 \mu \mathrm{m}$ feature has been examined in individual spectra of this source contained in the LRS database. None of the scans show anomalies at this wavelength or cosmic ray spikes. It is significant that the temperature of the blackbody that estimates the continuum of $15084-5701$ is 410 $K$, just the threshold temperature for hydrogen effusion in the

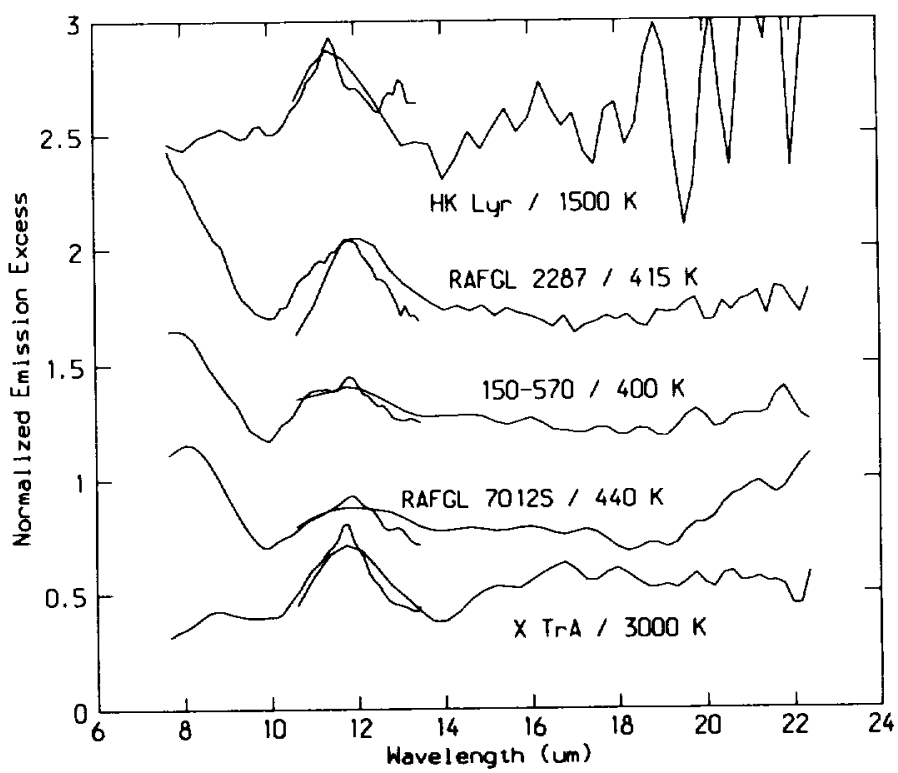

FIG. 4.-The average normalized emission excess spectrum of five carbon stars $08189-3602,15084-5702,18320-0352,18410+3654,18551+0323$. These stars appear to have narrow emission features in the 11.3 to $12.0 \mu \mathrm{m}$ region that are associated with unsaturated $\left(s p^{2}\right)$ bonds.

laboratory. When this star changes into a planetary nebula, then the $11.3 \mu \mathrm{m}$ band should become apparent. We propose this circumstellar grain evolution is analogous to the laboratory experiments on $\alpha: C-H$ by Dischler et al. (1983a, b).

Three other stars that are listed in Table 2 are interesting to note in regard to possible narrow PAH features: HK Lyr = $18410+3654,18551+0323$, and 15094-6953. 18320-0352 may be interesting but has low $\mathrm{S} / \mathrm{N}$. Their spectra are also shown in Figure 4. These spectra display peculiarly long central wavelengths of a narrow emission peak superimposed on the wide petestal. The narrow feature is not attributable to $\mathrm{SiC}$ or the typical PAH/UIR emission. Its width and center wavelength are variable form source to source. Both parameters fall within an acceptable range for interpretation similar to that discussed for $15084-5701$ in the preceeding paragraph. While there is an atomic line at $11.77 \mu \mathrm{m}$ due to $\mathrm{Cl} \mathrm{IV}$, it is not expected to be present in these objects because it requires a highly ionizing radiation. That radiation would produce other emission lines normally seen in planetary nebulae, that are not seen in these objects. Some of these objects belong to the coldest classes of carbon stars, and yet only a few objects from these classes display such features. Apparently low temperatures are required for the formation of PAH molecules, as well as some other unique circumstances that are as yet undetermined.

TABLE 2

Peculiar Narrow $11+\mu$ m Bands in Carbon Stars

\begin{tabular}{clcccc}
\hline \hline \multicolumn{1}{c}{ IRAS Name } & Other Name & ACII & $\begin{array}{c}\text { LRS } \\
\text { AutoClass }\end{array}$ & $\begin{array}{c}\text { LRS } \\
\text { Class }\end{array}$ & $\begin{array}{c}11+\mu \mathrm{m} \\
\lambda_{\text {eentor }}\end{array}$ \\
\hline $15084-5701 \ldots \ldots \ldots$ & \multicolumn{1}{c}{$\ldots$} & 5 & $\lambda 20: 1$ & 42 & 11.85 \\
$15094-6953 \ldots \ldots \ldots \ldots$ & X TrA & 3 & $\lambda 25$ & 42 & 11.73 \\
$18320-0352 \ldots \ldots \ldots \ldots$ & RAFGL 7012S & 5 & $\lambda 20: 1$ & 42 & 11.85 \\
$18410+3654 \ldots \ldots \ldots$ & HK Lyr & 3 & $\lambda 25$ & 42 & 11.43 \\
$18551+0323 \ldots \ldots \ldots$ & RAFGL 2287 & 5 & $\lambda 20: 1$ & 42 & 11.85 \\
\hline
\end{tabular}


The $11.85 \mu \mathrm{m}$ transition of PAHs is due to the $\mathrm{C}-\mathrm{H}$ out-ofplane deformation mode of saturated edge sites. With an sufficiently cold carbon star, the $11.85 \mu \mathrm{m}$ band ought to be apparent if these ring molecules are present and abundant. Its appearance at a temperatures less than $4000 \mathrm{~K}$ would indicate that $\alpha: C-H$ edge sites become fully saturated below this temperature. Heating studies of $\alpha: \mathrm{C}-\mathrm{H}$ samples in the laboratory that are made from radio frequency plasma deposited benzene on fused quartz and germanium substrates demonstrate the evolution of the unsaturated edge sites $\left(s p^{3}\right)$ to saturated sites ( $s p^{2}$ either olefinic or aromatic; Dischler et al. $1983 \mathrm{a}, \mathrm{b}$ ) that is attributable to hydrogen effusion at temperatures above $400 \mathrm{~K}$. The concentrations of these sites are modified by thermal annealing in two separate temperature regimes: $450-600 \mathrm{~K}$ and $600-900 \mathrm{~K}$. Up to $600 \mathrm{~K}$, the $s p^{3} / s p^{2}$ ratio increases indicating the removal of $s p^{3}$ unsaturated bonds by hydrogen attachment and cross-linking. In the $600-900 \mathrm{~K}$ region, hydrogen is effused until at $900 \mathrm{~K}$, all the carbon atoms have $s p^{2}$ aromatic hybridization. Such hydrogen effusion would manifest itself spectrally through an evolution of $\mathrm{C}-\mathrm{H}$ emission features in an appropriately chosen sequence of objects. In the $11 \mu \mathrm{m}$ region, the sequence is ordered from $\sim 12 \mu \mathrm{m}$ into 11.3 $\mu \mathrm{m}$ features. The annealing process is not reversible in temperature, and implies that the starting material $s p^{3}$ (unsaturated bonds) is created at low temperatures and subsequently becomes $s p^{2}$ (saturated bonds). This sequence is in line with the supposed evolution of carbon stars into planetary nebular. It is not completely analogous to the induced nucleation theory proposed by Frenklach et al. (1989).

We can conclude that when PAH or HACs are present in classical carbon stars, and they emit a narrow feature in the 11 $\mu \mathrm{m}$ region, it occurs at about $11.9 \mu \mathrm{m}$, not $11.3 \mu \mathrm{m}$. In environments where copious UV radiation is present, they emit at 11.3 $\mu \mathrm{m}$, not $11.9 \mu \mathrm{m}$. The evolution of the feature from 11.9 to 11.3 $\mu \mathrm{m}$ reflects the effusion of hydrogen from the starting material, and the evolution is a result of thermal processing of the material. Material with unsaturated bonds evolves into that with saturated bonds, either from thermal or UV radiation induced heating. It is not necessary for PAHs to be generated in carbon star atmospheres and supplied directly to the interstellar medium (ISM). HACs in the ISM can be modified under the influences of UV radiation when their temperatures are elevated over the threshold of hydrogen effusion, $600 \mathrm{~K}$, by absorption of UV photons.

Pure carbon dust with $\mathrm{C}-\mathrm{C}$ and $\mathrm{C}=\mathrm{C}$ bonds was not found. Graphite lacks identifiable bands in the LRS range. $C_{60}$ displays two prominent bands at 17.55 and $19.3 \mu \mathrm{m}$ (Onari, Tada, \& Toshihiro 1991) that are not observed in any of the Class spectra or the individual object spectra.

Below $500 \mathrm{~K}$, an emission at $\lambda>20 \mu \mathrm{m}$ is noticeable in the spectra of Classes 1 and 5. The feature is the blue end of the 30 $\mu \mathrm{m}$ feature that has been observed in carbon stars and planetary nebulae (Forrest et al. 1981; Goebel \& Moseley 1985) and has been associated with MgS (Goebel 1980; Goebel \& Moseley 1985; Nuth et al. 1985). This rise in emission is not to be confused with the $21 \mu \mathrm{m}$ emission (Kwok, Volk, \& Hrivnak 1989) found in certain carbon stars with bright visual components, and associated with $\mathrm{SiS}_{2}$ by Goebel (1993). This emission at $\lambda>20 \mu \mathrm{m}$ is noticeable in the LRS spectra of individual stars, e.g., IRC +10216 , in the Classes in which the MgS band has already been observed and is not an artifact of detector saturation, data processing problems, or erroneous calibrations. In Classes 1 and 5 we find an extensive observing list of stars that should be valuable to future airborne or satellite observing programs in the far infrared.

The broadband colors of Classes 1 and 5 display the expected characteristics of the $\mathrm{MgS}$ band. In the [12]-[25], $[25]-[60]$ color-color diagram, the Class mean color is shifted to the right, and in the $[25]-[60],[60]-[100]$ diagram the Class mean color is negative. This negative color can be interpreted as a color deficiency at $100 \mu \mathrm{m}$, i.e., as an emission feature that shows an excess in the 25 and $60 \mu \mathrm{m}$ bands, and which returns to the continuum at $100 \mu \mathrm{m}$. The mean colors of Classes 1 and 5 are strikingly different than those of Class 0 , which includes much hotter stars, and Class 6 , which contains hot stars with significantly positive $[60]-[100]$ colors (emission excesses) that can be interpreted as cold shells of carbon grains. Therefore, the colors of Classes 1 and 5 are not consistent with cool circumstellar shells of pure carbon grains.

\subsection{Evolution}

The observed correlation of Class temperature with the presence of the $\mathrm{SiC}$ and $\alpha: \mathrm{C}-\mathrm{H}$ dust species suggests a grain condensation sequence in which $\mathrm{SiC}$, the most refractory of carbon rich dust species, condenses out of the atmosphere first. As the atmosphere evolves, cools and expands, SiC forms the condensation nuclei for the subsequent formation of $\alpha: \mathrm{C}-\mathrm{H}$ (Martin \& Rogers 1987). At still lower temperatures and later times, MgS catalysis occurs on the grains (Goebel \& Moseley 1985). Although the evolution of carbon stars is not clearly understood, this condensation sequence should occur in appropriate carbon stars whose circumstellar shells evolve from higher to lower temperatures. From the statistics of group membership in the Classes included in the sequence shown in Figure 1, roughly $\frac{1}{2}(60 \%)$ of all the carbon stars that are included in this study can be identified as falling into this evolutionary sequence, There are a substantial number of spectra that do not fit into this sequence and display molecular absorption features, specifically, Class 3 .

It is significant that, as carbon stars are thought to be the red giant evolutionary phase that precedes the protoplanetary and planetary nebula phases, the spectral signatures of $\alpha: C-H$ are found at an earlier evolutionary phase than are the spectral signatures of PAHs. Although not included in the carbon stars studied here, the prototypical carbon rich protoplanetary nebula $04395+3601=$ PK $166-6.1=$ RAFGL 618 (MetaClass $\gamma 0$ ) displays the broad absorption features without the narrow $11.3 \mu \mathrm{m}$ emission, and yet has weak narrow PAH type features at $3.3 \mu \mathrm{m}$. The presence of the narrow emission features at $3.3 \mu \mathrm{m}$ may be the result of nacient bipolar nebula formation in this object, or they may be $\alpha: C-H$ emission similar to that observed by Dischler et al. $(1983 \mathrm{a}, \mathrm{b})$ in the laboratory. Since the dust temperature of RAFGL 618 is very low, $\sim 150 \mathrm{~K}$, it is unlikely that the PAHs exist as free molecules, but rather have condensed completely into the solid phase. The vapor pressure of a heavy molecule like a PAH at $150 \mathrm{~K}$ is absolutely negligible, and there is no evidence that PAHs sublimate. So before the planetary nebulae phase begins, the balance in the population of species should be dramatically in favor of simple $\mathrm{C}-\mathrm{O}$ molecules, and not PAHs. As the planetary nebula develops, excitation of PAH emissions should progress and eventually dominate the spectrum. As an aside remark, Jourdain de Muizon et al. (1990) suggest that CRL 618 displays a silicate emission spectrum in the LRS range of wavelengths. We interpret the spectrum as displaying absorption bands of the molecules $\mathrm{HCN}$ and $\mathrm{C}_{2} \mathrm{H}_{2}$ centered at 
7 and $13 \mu \mathrm{m}$. CRL 618 is engaged in form of spectral mimicry, of which more will be said later. It is not the sole example of this phenomenon; many such objects are found in Class 3 .

Confirmation for this line of reasoning is found by considering the spectra of the LRS 92 objects $08189-3602=$ PK $245+0.1$, and $12063-6259=\mathrm{He} 2-77=\mathrm{PK} \quad 298-0.1=$ RAFGL 4144 (Fig. 5), members of the $\gamma 0$ MetaClass, and of the same split Class $\gamma 0: 2$ as RAFGL 618 . All have a similar $T_{\mathfrak{c}} \sim$ $180 \mathrm{~K}$, but $08189-3602$ and $12063-6259$ have a weak 11.6 $\mu \mathrm{m}$ narrow features on a broad $11+\mu \mathrm{m}$ pedestal along with strong 8.5 and $7.7 \mu \mathrm{m}$ emissions, as well as emission lines at $12.9,15.5$, and $18.8 \mu \mathrm{m}$, due to, respectively, Ne II, Ne III, and S III (Jourdain de Muizon et al. 1990). The latter two objects are probably not planetaries, rather they are most likely $\mathrm{H}$ II regions (Simpson \& Rubin 1990). They are too luminous by 1 or 2 orders of magnitude based on their radial velocities and Galactic latitudes. They are relevant to the present discussion by virtue of their displaying unusually long central wavelengths for their narrow $11+\mu \mathrm{m}$ emissions. Probably, PK $245+0.1$ and $298-0.1$ have just started to dissipate their shells, and the $11.6 \mu \mathrm{m}$ band should in time shift into an 11.3 $\mu \mathrm{m}$ band. The dissipation process for protoplanetaries and young $\mathrm{H}$ Il regions is probably very similar, with the details being controlled by the luminosity of the central source and its temperature. The lower limit to protoplanetary shell temperatures of carbon-rich objects prior to the onset of the planetary nebula eruption, e.g., emergence of the bipolar emitting regions that are presumably in the polar regions of the circumstellar shell, is $\sim 150 \mathrm{~K}$. RAFGL 618 could exemplify the circumstellar shell just prior to eruption. Kwok \& Feldman (1978) observed RAFGL 618 in free-free emission at $6 \mathrm{~cm}$ and classified its central source as B2. The transition spectral type may be between B2 and 07 (A. G. G. M. Tielens 1994, private communication). See Martín-Pintado et al. (1992) for a schematic of RAFGL 618 deduced for $\mathrm{NH}_{3}$ observations that is in line with this interpretation.

Do our results imply an evolutionary scheme significantly different from previous proposals, or is an alternative scheme

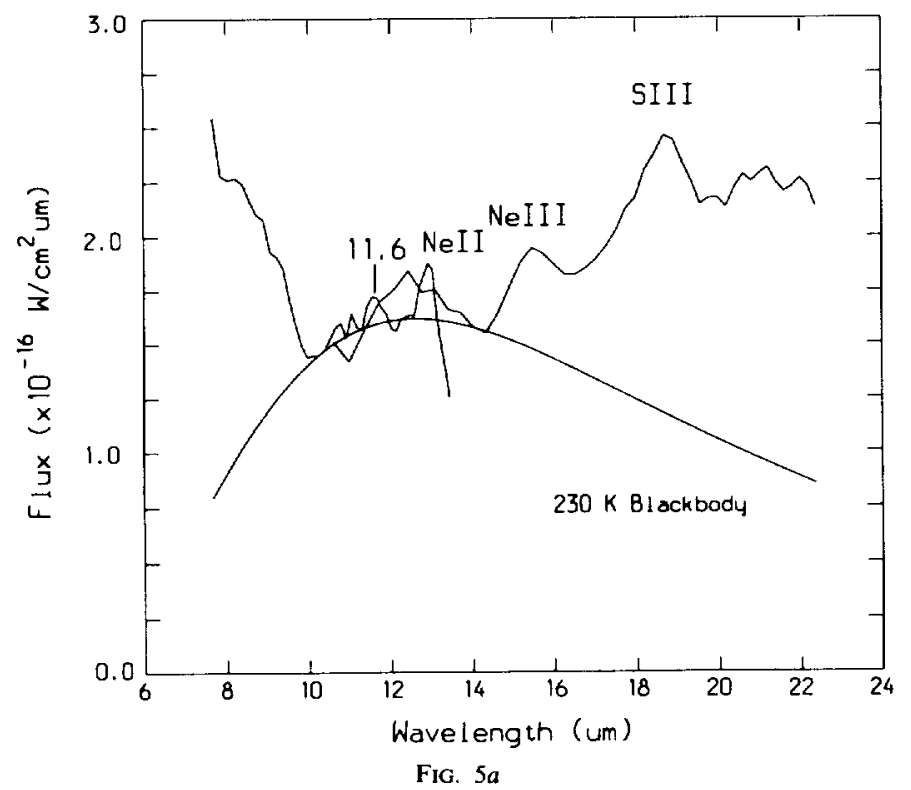

required? We are able to fit a large fraction of the carbon stars, those with circumstellar emission features, into a continuum temperature sequence. What we can say is this: If carbon stars evolve to lower temperatures and larger $\mathrm{C} / \mathrm{O}$, then the sequence of LRS spectral types in Figure I can be interpreted to represent an evolution in the circumstellar dust shell from $\alpha-\mathrm{SiC}$ to $\alpha: \mathrm{C}-\mathrm{H}$ that makes physical sense. Due to the large number of free parameters, there are always exceptions to the rules. The crucial parameter $L_{*}$ is not well-determined for carbon stars of any class. Until their distances are determined reliably, $L_{*}$ and $M_{*}$ influences on the sequence in temperature will remain an open question. Because of this uncertainty, our simple sequence in temperature remains open to interpretation, yet the sequence remains established.

We note that the LRS spectra alone are insufficient to disentangle the basic stellar parameters $T_{\text {eff }}, \mathrm{C} / \mathrm{O}, M_{*}, L_{*}$, etc. There have been recent studies examining the masses of circumstellar envelopes of carbon stars and distances derived. The study of Olofsson et al. (1993) summarizes the available data for some of the stars $(\sim 120)$ in the present study. In order to arrive at distances, an absolute magnitude in the $K$ band of -8.1 was assumed. If all the stars have the same absolute luminosity, then the sequence in temperature is easily converted into evolution. However, that assumption of similarity is suspect when applied to our larger sample.

Chan and Kwok's evolutionary scheme can be stated simply as follows: Most carbon stars are formed before they begin to form dust shells. Thereafter, they evolve to lower temperatures and thicker $\mathrm{SiC}$ dust shells. The driving mechanism here is an increasing $\mathrm{C} / \mathrm{O}$ that is the result of helium shell flashes in the interior and plumes that mix the triple- $\alpha$ generated material to the surface, where it is driven outward into the circumstellar regions by a stellar wind. Chan and Kwok's conclusions regarding the evolution of carbon stars is based largely on their comparison of radiative transfer models with the photometry in the literature that ranges from near to far infrared, a technique similar to that employed previously by RowanRobinson \& Harris (1982, 1983), van der Veen \& Habing

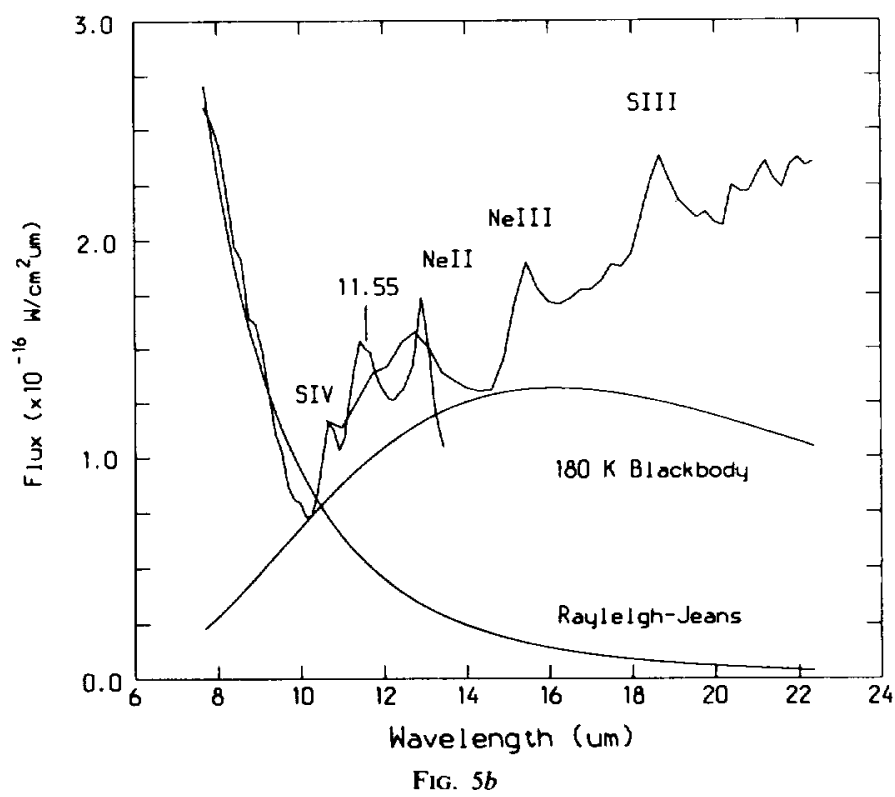

FIG. 5.-Spectra of the H Il regions $08189-3602(a)$ and $12063-6259(b)$. These objects appear to have narrow emission features at $11.6 \mu \mathrm{m}$, rather than the nominal value of $11.3 \mu \mathrm{m}$ associated with the UIR/PAH series of features. 
(1988), and Willems \& de Jong (1986a, b). The latter reached somewhat different conclusions regarding the evolution of carbon stars while employing similar data sets and models.

Willems and Jong's scenario is this: Most carbon stars are formed after dust shell formation. A further increase in $\mathrm{C} / \mathrm{O}$ produces an evolutionary sequence in dust population in the shell starting at silicates and progressing to $\mathrm{SiC}$ and carbon. The scheme of Willems and de Jong recognizes the population of Class 11 and wrestles with the consequences. Chan and Kwok (as well as Zuckerman 1993) dismiss Class 11's characteristics as extraneous or spurious. This difference largely accounts for the orthogonal viewpoints each group achieves with essentially the same starting data and analysis approach.

Here, the evolutionary scenario for the dust outlined differs significantly from that proposed by Chan \& Kwok (1990). They studied the circumstellar emission spectra of many of the same objects and concluded that the evolution of $\mathrm{SiC}$ from hexagonal-rhombohedric form, $\alpha-\mathrm{SiC}$, to the cubic form, $\beta$-SiC, occurs at a transition temperature $\sim 2000 \mathrm{~K}$, and that the $8.7 \mu \mathrm{m}$ emission feature is due to compound carbon molecular bands near the photosphere. They chose to describe the dust opacity throughout the infrared as "empirical SiC" in spite of the large body of research that had clearly shown that separate carbon and $\mathrm{SiC}$ populations are the appropriate description of the circumstellar dust. This distinction is more than mere semantics. The distinction between the two grain populations leads to different possibilities for describing not only the spectra, but also the evolutionary history of the shells and stars within them. The empirical $\mathrm{SiC}$ dust is distinctly different from the $\mathrm{Si}$ polluted carbon dust discussed by Martin \& Rogers (1987), in that the latter considered the carbon dust to have occasional $\mathrm{Si}-\mathrm{C}$ bonds incorporated in the bulk that gave rise to the $\mathrm{SiC}$ feature, while the $\mathrm{C}-\mathrm{C}$ bond complexes, whatever their exact form, produced a featureless continuum throughout the infrared. Carbon dust is well-founded theoretically and observationally, especially for the infrared bright carbon star IRC + 10216 (see Gail \& Sedlmayr 1987). Chan and $\mathrm{K}$ wok would have been better served by naming their opacity empirical carbon star dust rather than empirical SiC. The recent article by Lorenz-Martins \& Lefèvre (1993) thoroughly discusses the multicomponent dust model through radiative transfer calculations and compares the results with observations. The weight of evidence is clearly on the side of multiple dust components.

Our Class 11, those stars that display silicate like emission, or its equivalent in other studies, is at the center of the debate in the literature relating to the evolutionary paths of carbonrich stars and their circumstellar shells (Zuckerman \& Maddalena 1989; Zuckerman 1993; Willems \& de Jong 1988; de Jong 1989; van der Veen \& Habing 1988). The resolution of the debate must explain Class 11 , not ignore it. It is outside the scope of this work to address this issue beyond pointing out that Class 11 will not go away and hide; it demands explanation. We do point out the possible role of spectral mimicry apparent in some carbon star spectra, such as $\mathrm{T}$ Lyr and AFGL 618. The spectra of some, but not all, of the objects in Class 11 are attributable to this mechanism.

We suggest that our spectral sequence based on $T_{\mathrm{C}}$ mimics an evolutionary sequence in time and/or mass paralleling an increasing $\mathrm{C} / \mathrm{O}$. If the evolution of carbon stars from visually bright to infrared bright is indeed correct, then the evolution must be toward increasing $\mathrm{C} / \mathrm{O}$. We consider the set of LRS spectra to reflect evolution in time of carbon stars and to rep- resent the various states they would possess if sampled randomly. Then we can consider the progression of spectral types in Figure 1 to recapitulate the evolution of such stars. This interpretation is analogous to the tranditional spectral classification sequence in visible astronomy that uses a twodimensional parameter set consisting of temperature and spectral feature strength. We propose that such a two-dimensional parameterization also applies to dust features in the infrared spectra of carbon stars and is a useful classification methodology. The two parameters are $T_{\mathrm{C}}$ and feature strength of the $\mathrm{SiC}$ and $\alpha: \mathrm{C}-\mathrm{H}$ components. Because broadband color-color diagrams are not very sensitive to the feature differences, the use of these diagrams does not produce a unique mapping into these two parameters, so it is necessary to utilize spectrophotometry as is present in the LRS. A narrowband filter set specifically tuned to the dust features would give useful information. The selection of such a set would require careful consideration due to the presence of Class 3 stars in any random sample of carbon stars.

\subsection{Dust Condensation and Mass Loss}

Homogeneous dust condensation theories require carbon dust to nucleate on preexisting molecules and cluster complexes, not on condensation nuclei like $\mathrm{SiC}$ or TiC. From homogeneous condensation calculations, it has become well known that $\mathrm{SiC}$ condenses at a higher temperature than carbon dust (Gilman 1969; McCabe 1982). Class 4 is consistent with homogeneous condensation of $\mathrm{SiC}$. Various types of carbon dust (graphite, amorphous carbon, tetrahedrally coordinated carbon, hydrogenated amorphous carbon, diamonds, etc.) are considered to form depending upon a variety of initial conditions. There are identifiable instances where homogeneous nucleation of carbon dust is consistent with the observations, such as the hydrogen deficient R CrB stars. Because the chemistry of hydrocarbons is very complex, there have been a wide range of proposals as to the precise molecular pathway $\left(C_{3}\right.$, $\mathrm{C}_{2} \mathrm{H}_{2}$, PAH, etc.) from which the dust $(\alpha: \mathrm{C}-\mathrm{H}$, graphite, $\mathrm{HAC}$, amorphous or glassy carbon) forms. The red giant phase should be warm enough $\left(1500<T_{e}<3000 \mathrm{~K}\right)$ to allow diverse populations of molecules and dust as has been modeled by Gail \& Sedlmayr (1987). Their models include PAHs in a thermodynamically natural manner. For most carbon stars in our study, we have found that homogeneous dust populations are not the rule, and heterogeneous populations predominate.

From other studies there is mounting evidence that dust condensation in carbon rich circumstellar shells proceeds heterogeneously. Studies by McCabe (1982), using equilibrium nucleation theory, Gail \& Sedlmayr (1984) and Frenklach et al. (1989) conclude that $\mathrm{SiC}$ condenses out closer to the photosphere and at higher temperatures than does amorphous carbon dust. Hence, as mass is driven outward from the photosphere deeper into the circumstellar shell by the stellar wind, where lower temperatures prevail, $\mathrm{SiC}$ is injected as readily available nuclei or seedlings for the heterogenous condensation of carbon. This scenario is consistent with our interpretation of the class spectra and needs theoretical confirmation in regard to the essential details. While it is clear that there should be spectral changes due to the incorporation of various species, one might suspect structural differences in the circumstellar shells. In the models, the resulting global thermodynamic and hydrodynamic shell structure, appears not to be so much dependent on the particular dust species, but rather the fact that dust is present or absent (Gail \& Sedlmayr 1987; Fleischer, 
Gauger, \& Sedlmayr 1992; Winters, Dominik, \& Sedlmayr 1994).

Heterogenous condensation may have implications for the explanation of certain anomalous gas phase molecular abundances. Significant amounts of Si can be consumed by $\mathrm{SiC}$. Observations of Goldhaber \& Betz (1984) and Keady \& Ridgway (1993) point to an anomalous underabundance of silane inside $40 R_{*}$ that is unexplainable in terms of equilibrium or nonequilibrium gas phase chemistry in or near the photosphere. The Berlin group models show that condensation of carbon dust in carbon rich atmospheres usually proceeds through $\mathrm{C}_{2} \mathrm{H}_{2}$ or $\mathrm{C}_{2} \mathrm{H}$ implying hydrogenated carbon of some form is a likely condensate (see also Keady \& Hinkle 1988). $\mathrm{HAC}$ mantles on $\mathrm{SiC}$ grains could make the Si unavailable for gas phase or grain surface chemistry. This is an interesting area for further theoretical work.

The recent study of Lorenz-Martins \& Lefèvre (1993) examines the composition of a two component dust with radiative transfer models and compares the resultant flux curves with nine carbon stars. They find a range for the number density of grain populations, $N_{\mathrm{SiC}} / N_{\mathrm{AC}}$ of 0.03 to 0.20 , and support the conclusion of Skinner \& Whitmore $(1988 \mathrm{a}, \mathrm{b})$ that the strength of the $\mathrm{SiC}$ feature is related to the total mass-loss rate. Skinner \& Whitmore $(1988 \mathrm{a}$, b) related the strength of the $\mathrm{SiC}$ emission, $P(\mathrm{SiC})$, expressed as the product of the band emission to continuum ratio, the $I R A S$ band $I$ emission intensity, and distance squared of the star, directly to the $\mathrm{CO}$ radio emission line intensity and thereby the mass-loss rate, $d M / d t$. LorenzMartins \& Lefèvre (1993) related optical depth, $\tau_{\text {exl }}$, and $N_{\mathrm{sic}} / N_{\mathrm{Ac}}$. All four parameters appear to be related to the mass loss rate in the circumstellar shell. The correlation between $\tau_{\text {ext }}$, $N_{\text {SiC }} / N_{\text {AC }}, P(\mathrm{SiC})$, and $\mathrm{CO}$ integrated line intensity appears to be positive, allowing for a few exceptional stars like RY Dra. Neither Skinner \& Whitmore (1988a, b) or Lorenz-Martins \& Lefèvre (1993) recognized the difference between the $\mathrm{SiC}$ band and the $\alpha: \mathrm{C}-\mathrm{H}$ band, nor that of the "windowing effect" of molecular absorption bands seen against hot continua, a coincidence of absorption features that we refer to a spectral mimicry. The distinction does not invalidate their result; rather, it may account for some of the dispersion in their measures. For example RY Dra is a member of carbon star Class 3, a Class that is dominated by hot continua with molecular bands in absorption. By shear coincidence, spectral mimicry, it happens that the RY Dra emission feature is the window between two HCN absorption bands (see Fig. 6). It is not really valid to place it in Figure 12 of Lorenz-Martins \& Lefèvre (1993) that correlates $\tau_{\mathrm{ext}}$ and $N_{\mathrm{Sic}} / N_{\mathrm{AC}}$. Extraction of RY Dra improves the correlation between those two parameters. In Figure 6, the 1 to $25 \mu \mathrm{m}$ infrared spectrum of RY Dra is shown to exemplify the spectral mimicry concept. For similar reasons, the exclusion of Y CVn from the study of Skinner \& Whitmore $(1988 \mathrm{a}, \mathrm{b})$ could improve the scatter in their relationships. In fact, all Class 3 objects should be carefully considered in such studies to avoid confusion by spectral mimicry.

Although Lorenz-Martins \& Lefèvre (1993) did not use core mantle grains in their study, they favor an interpretation of the data in such terms, and promised a future contribution relating to it. Such heterogenous nucleation has been favored by several investigators of carbon star dust condensation (e.g., McCabe 1982; Frenklach et al. 1989). However, the theory is most commonly applied to the condensation of ice mantles in dark clouds (e.g., Tielens \& Allamandola 1987), and the results are not directly applicable to the conditions in carbon star circumstellar shells.

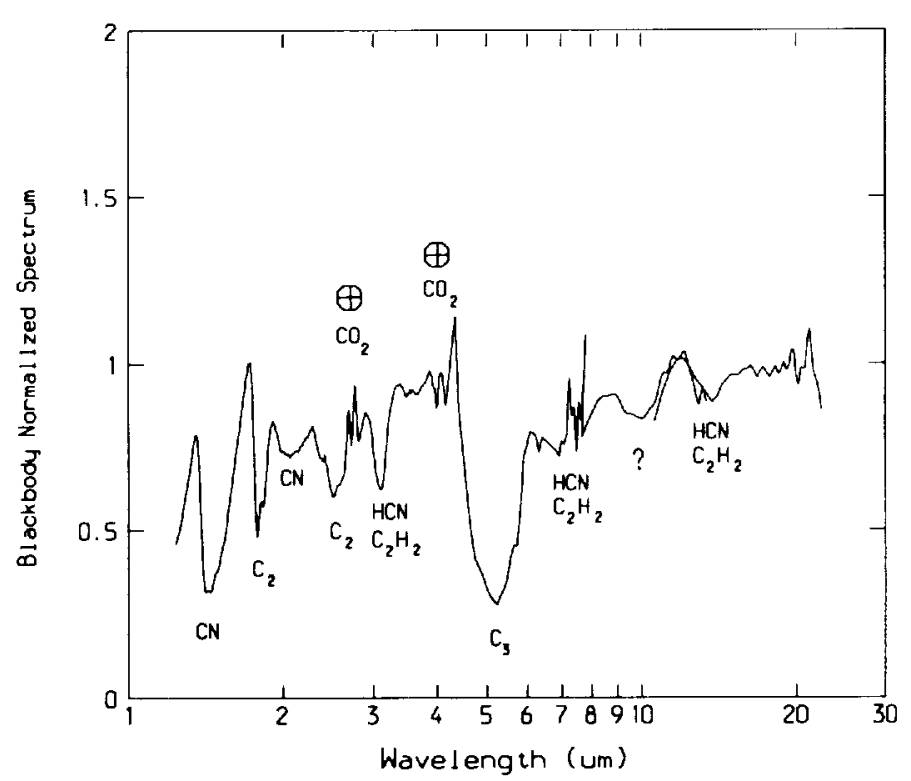

Fig. 6- The normalized emission excess spectrum of the carbon star RY Dra between 1.2 and $24 \mu \mathrm{m}$. This figure combines spectra taken from NASA's Kuiper Airborne Observatory and NASA's IRAS mission. The spectra were obtained on the dates: $1.2-5.6 \mu \mathrm{m}, 1976$ December $14 ; 4-8 \mu \mathrm{m}, 1979$ April 10; 8-24 $\mu \mathrm{m}$; 1983 January-November. Continuum removal included the contributions of the photosphere and a $300 \mathrm{~K}$ amorphous carbon shell.

Whatever their origins, the strengths $11+\mu \mathrm{m}$ emission features appear to be related to the total mass-loss rate, as long as the features are optically thin. As the features express the abundances of different dust populations, the exact nature of the correlation is in need of revision. There are probably two tracks in Figure 1 of Skinner \& Whitmore (1988a, b), one for pure $\mathrm{SiC}$, corresponding to Class 4 , and the other for pure $\alpha: \mathrm{C}-\mathrm{H}$, corresponding to Class 5 , as well as a continuum of mixed states, corresponding to Classes 0 and 1.

We anticipate that the $\mathrm{SiC}$ emitting stars, Class 4 , should show a lower mass-loss rate than the $\alpha: C-H$ stars, Class 5 , because the latter tend to be the infrared bright carbon stars, and the former the visually bright carbon stars. Indeed, there are no members of Class 4 in the sample of Skinner \& Whitmore (1988), indicating a lack of observable $\mathrm{CO}$ radio emission lines from which distances were estimated. There are 10 members of Class 0, i.e., those with mixed $\mathrm{SiC}$ and $\alpha: \mathrm{C}-\mathrm{H}$ and continua temperatures averaging $800 \mathrm{~K}$. These 10 members of Class 0 that are common to both studies have an average of $d M / d t \sim 4.2 \times 10^{-6} M_{\odot} \mathrm{yr}^{-1}$. The values are dispersed over 2 orders of magnitude. The two members of Class 5 are IRC +10216 and CRL 2155 , both of which exceed $d M / d t \sim$ $2 \times 10^{-5} M_{\odot} \mathrm{yr}^{-1}$.

The Class 3 stars, also visually bright, should show measurable mass-loss rates. The dust-to-gas mass must be measured by an alternate procedure that takes into account the strength of the continuum of an emitting dust shell. This must be estimated by subtraction of a photosphere, after careful consideration of the effects of molecular features. Indeed there are very few stars for which there is available sufficient spectrophotometric data spanning the 1 to $30 \mu \mathrm{m}$ spectral region for the conduct of such a study. The current authors are preparing such a compilation using aircraft and satellite data. The spectrum of RY Dra shown in Figure 6 is an example. The members of Class 3 in the sample of objects used by Skinner \& 
Whitmore $1988 \mathrm{a}$, b) have consistently low $d M / d t \sim 1.9 \times 10^{-7}$ $M_{\odot} \mathrm{yr}^{-1}$. CIT 6, not included in this estimate, is atypical of the sample of seven sources and has a $d M / d t \sim 3.2 \times 10^{-6} M_{\odot}$ $\mathrm{yr}^{-1}$. It may have classification problems induced into its spectrum by the nonlinear detector effects of extremely bright sources. IRC +10216 clearly has this problem.

Based upon this small sample of common objects in the two studies, it appears that the division of spectra and association of spectral features proposed here is reflected in the mass-loss rates of the objects as measured by Skinner \& Whitmore (1988a, b). Generally, circumstellar shells with colder temperatures display $\alpha: \mathrm{C}-\mathrm{H}$ and have higher mass-loss rates; warmer circumstellar shells display $\mathrm{SiC}$ and have lower massloss rates. There are two notable exceptions, CIT 6 and IRC +10216 . Exceptions are to be expected in any study of carbon stars. Hopefully improvements in radio receivers will result in sufficient sensitivity for the measurement of distances necessary to this study. A further study of the entire sample of carbon stars in the LRS with these more sensitive radio receivers ought to clarify this division.

The combination of homogeneous condensation of seedlings and heterogeneous condensation of mantles has been termed induced nucleation by Cadwell et al. (1994). Their model elaborates the proposition by Frenklach et al. (1989) that a circumstellar shell surrounding a carbon-rich red giant star has multiple zones in which condensation takes place homogeneously nearest the star, and heterogeneously for amorphous carbon and PAH condensates in more extended zones. The discussion of our spectral sequence lends credence to such a scheme by identifying Classes of carbon star spectra consistent with such zones. Class 4 has the hottest continuum temperature, $3000 \mathrm{~K}$, and shows exclusively $\mathrm{SiC}$ emission. Class 0 , the next hottest at $800 \mathrm{~K}$, shows the beginnings of the $8+\mu \mathrm{m}$ $\alpha: C-H$ band and the $\mathrm{SiC}$ band combined with the $11+\mu \mathrm{m}$ $\alpha: \mathrm{C}-\mathrm{H}$ band. Classes 1 and 5 are progressively colder, 600 and $470 \mathrm{~K}$, and exhibit stronger $\alpha: \mathrm{C}-\mathrm{H}$ bands, and little to none of the $\mathrm{SiC}$ band. Finally, two objects, 15804-5701 and $08189-3602$, exhibit unsaturated $\left(s p^{3}\right)$ hydrocarbon emission bands at 11.85 and $11.6 \mu \mathrm{m}$ and very low continuum temperatures of 410 and $150 \mathrm{~K}$.

We could consider the spectra to sample progressively colder regions of the circumstellar shell as those regions approach optical depth one progressively farther from the photosphere. The temperatures noted in Figure 1 do not agree precisely with those quoted by Frenklach et al. (1989) in their schematic diagram of the circumstellar shell's dust forming regions. Our temperatures are continuum emission temperatures, and may not accurately reflect the conditions in the formation region for a particular species, but rather that of the dominant emitting region underneath. A detailed radiative transfer computation is required to associate radiation temperatures with features in the emitting spectra. In spite of this, the sequence of species deduced is in agreement with their schematic.

Heterogenous nucleation, or induced nucleation, or catalysis is almost certainly required to explain the presence of $\mathrm{MgS}$ and $\mathrm{SiS}_{2}$ emission features in many carbon-rich circumstellar shells (Goebel \& Moseley 1985; Nuth et al. 1985; \& Goebel 1993). Because of the relatively low abundances of the sulfides compared with carbon, nearly all the sulfur in the coldest regions of the shells must condense out onto relatively large grains. This is only possible on preexisting grains of carbon through, for example, an efficient catalytic reaction. It is worthwhile to note that although there are many instances of $\mathrm{MgS}$ band ( $30 \mu \mathrm{m})$ indicated in the long-wavelength spectra of the carbon stars in our sample, there are no occurrences of the more easily perceived $\mathrm{SiS}_{2}$ band $(21 \mu \mathrm{m})$. If the $21 \mu \mathrm{m}$ band were due to the presence of some hypothetical carbon compound, we find its lack of occurrence difficult to explain.

Heterogenous nucleation, or induced nucleation with $\mathrm{SiC}$ seedlings, is limited in its application to carbon stars. It cannot be invoked to explain the condensation process in hydrogen deficient $\mathrm{R} \mathrm{CrB}$ type stars. They show no $\mathrm{SiC}$ bands, so the nucleation must be homogenous, at least in the sense that only carbon compounds are involved. These stars are thought to have much higher surface gravity and therefore, gas densities, a situation that enhances comparatively three-body collisions, a theoretical barrier to homogeneous nucleation theory.

\section{CONCLUSIONS}

A new classification scheme of the IRAS LRS carbon stars has been presented. It comprises the separation of 718 probable carbon stars into 12 distinct self-similar spectral groupings. Continuum temperatures are assigned and range from $470 \mathrm{~K}$ to $5000 \mathrm{~K}$. Three distinct dust species are identifiable: $\mathrm{SiC}, \alpha: \mathrm{C}-\mathrm{H}$, and $\mathrm{MgS}$. SiC and $\alpha: \mathrm{C}-\mathrm{H}$ band strengths are found to correlate with the temperature progression among Classes. If temperature corresponds to evolution, then $\mathrm{SiC}$ grains precede $\alpha: C-H$ and form the nuclei for the condensation of the latter material. At still lower temperatures, $\mathrm{MgS}$ appears to be quite prevalent. We conclude that $\mathrm{SiC}$ seeding of the circumstellar gas is a prerequisite for the efficient growth of hydrocarbon grains. Heterogenous (or induced) nucleation appears to be a common, although not an universal, mechanism in carbon rich circumstellar shells. It is thermodynamically efficient and able to account for the multitude of species that give rise to the several $11+\mu \mathrm{m}$ spectral features apparent in these objects.

No $11.3 \mu \mathrm{m}$ PAH features are identified in any of the 718 carbon stars. However, a handful of objects, IRAS 15048 - 5702, display an $11.85 \mu \mathrm{m}$ emission feature characteristic of laboratory samples of coronene, a PAH. That feature corresponds to the $\mathrm{C}-\mathrm{H}$ vibrational waging of saturated edge sites. These few examples of narrow emissions between 11.3 and $12 \mu \mathrm{m}$ are noted and attributed to hydrocarbon bonds for the first time. The following puzzle presents itself. If $\alpha: \mathrm{C}-\mathrm{H}$ or HACs are developed from PAH molecular constituents, and these must be very numerous, then why are not the PAH narrow emission features seen in the spectra of any of the hundreds of carbon stars from the LRS Atlas? One UV photon corresponds energetically to about 100 each $11 \mu \mathrm{m}$ photons. Certainly, the density of PAHs in a circumstellar environment, must be more than 100 times greater than that in the interstellar medium. $\alpha: \mathrm{C}-\mathrm{H}$ condenses at higher temperatures than do the aromatic hydrocarbons (PAHs), and this fact may explain this situation.

The new classification scheme yields a progression of spectra which when combined with estimated temperature of the underlying continua, can be interpreted as an evolutionary progression in time or mass. A two-dimensional classification of temperature and feature strength can be extracted that corresponds to this evolution. It is vitally important to determine the distances to many carbon stars individually and reliably so that their luminosities can be determined. Only then can the influence of time and mass be determined on this evolutionary sequence. 
It is a pleasure to acknowledge discussions with Jan Simpson, Xander Tielens, and Jesse Bregman. Don Strecker, Fred Witteborn, and Jesse Bregman contributed to the acquisition of KAO data for RY Dra. John Stutz and Will Taylor were essential in programming the Symbolics computers in Lisp. Corrections and suggestions for improvement by the referee are greatly appreciated.

\section{REFERENCES}

Allamandola, L. J., Tielens, A. G. G. M., \& Baker, J. R. 1985, ApJ, 290, L25

Amari, S., Hoppe, P., Zinner, E., \& Lewis, R. S. 1992, ApJ, 394, L43

Atlas of Low-Resolution IRAS Spectra. 1986, IRAS Science Team, prepared by F. M. Olnon \& E. Raimond, A\&AS, 65,607

Baron, Y., de Muizon, M., Papoular, R., \& Pégourié, B. 1987, A\&A, 186, 27

Borghesi, A., Bussoletti, E., Colangeli, L., \& De Blasi, C. 1983, Infrared Phys., 23,321

Bregman, J. D., Allamandola, L. J., Tielens, A. G. G. M., Geballe, T. R., \& Witteborn, F. C. 1989, ApJ, 344, 791

Bregman, J. D., Goebel, J. H., \& Strecker, D. W. 1978, ApJ, 223, L45

Brown, L. E., \& Clayton, D. D. 1992, ApJ, 392, L79

Bubenzer, A., Dischler, B., Brandt, G., \& Koidl, P. 1983, J. Appl. Phys., 54, 4590

Buss, Jr., R. H., Tielens, A. G. G. M., Cohen, M., Werner, M. W., Bregman, J. D., \& Witteborn, F. C. 1993, ApJ, 415, 250

Cadwell, B. J., Wang, H., Feigelson, E. D., \& Frenklach, M. 1994, ApJ, 429, 285

Chan, S. J. \& K wok, S. 1988, ApJ, 334, 362 1990, A\&A, 237, 354

Cheeseman, P., Kelly, J., Self, M., Stutz, J., Taylor, W., \& Freeman, D. 1988 , Proc. Fifth Machine Learning Conference (San Francisco: Morgan Kaufman), 54

Cohen, M. 1979, MNRAS, 186, 837

Cohen, M. Walker, R. G. \& Witteborn, F. C. 1992, AJ, 104, 2030

de Jong, T. 1989, A\&A, 223, L23

Dischler, B., Bubenzer, A., \& Koidl, P. 1983a, Appl. Phys. Lett., 42, 636

1983b. Solid State Comm., 48, 103

Dorschner, J., Friedemann, C \& Gürtler, J. 1977a, Ap\&SS, 48, 305 1977 b Astron. Nachr 298,279

Fadeyev, Y. A. 1983, Ap\&SS, 95, 357

Fleischer, A. J., Gauger, A., \& Sedlmayr, E. 1992, A\&A, 266, 321

Forrest, W. J. 1974, Ph.D. thesis, Univ. California, San Diego

Forrest, W. J Gillett, F. C. \& Stein, W. A. 1975, ApJ, 195, 423

Forrest, W. J., McCarthy, J. R., \& Houck, J. F. 1981, ApJ, 248, 195

Frenklach, M., Cramer, C. S., \& Feigelson, E. D. 1989, Nature, 339, 196

Friedemann, C., Gürtler, J., Schmidt, R., \& Dorschner, J. 1981, Ap\&SS, 79, 405

Gail, H. P., \& Sedlmayr, E. 1984, A\&A, 132, 163 1987, A\&A, 171, 197

Gal, O., de Muizon, M., Papoular, R., \& Pégourié, B. 1987a, Circumstellar Matter, ed. I. Appenzellar \& C. Jordan (Dordrecht: Reidel), 223

1987b, A\&A, 183,29

Gallino R Raiteri, C M. Busso M. \& Matteucci, F. 1994, ApJ, 430, 858

Gilman, R. C. 1969 ApJ, 155, L185

Goldhaber, D. M., \& Betz, A. L. 1984, ApJ, 279, L55

Goebel, J. H. 1980, BAAS, 12,858

1982, unpublished

1987, in Polycyclic Aromatic Hydrocarbons and Astrophysics, ed

A. Léger et al. (Dordrecht: Reidel), 329 1993, A\&A, 278, 226

Goebel, J. H., et al. 1980, ApJ, 235, 104

Goebel, J. H. Bregman, J. D., Witteborn, F. C., Taylor, B. J., \& Willner, S. P. 1981, ApJ, 246, 455

Goebel, J., Cheeseman, P., Self, M., Stutz, J., Gebault, F., \& Taylor, W. 1988, BAAS, 20,4

Goebel, J. H., \& Gerbault, F. 1988, in IAU Symp. 135, Proc. Interstellar Dust,

ed. Allamandola \& A. G. G. M. Tielens (NASA CP 3036), 519

Goebel, J. H., \& Moseley, S. H. 1985, ApJ, 290, L35
Goebel, J., Volk, K., Walker, H., Gebault, F., Cheeseman, P., Self, M., Stutz, M., \& Taylor, W. 1989, A\&A, 222, L5

Hartmann, L., \& Apruzese, J. P. 1976, ApJ, 203, 610

Hoare, M. G. 1990, MNRAS, 244, 193

Hoppe, P. Amari, S., Zinner, E., Ireland, T., \& Lewis, R. S. 1994, ApJ, 430, 870

Jourdain de Muizon, M., Cox, P., \& Lequeux, J. 1990, A\&AS, 83, 337

Keady, J. J., \& Hinkle, K. H. 1988, ApJ, 331, 539

Keady, J. J., \& Ridgway, S. T. 1993 , ApJ, 406, 199

Kwok, S., \& Feldman, P. A. 1978, ApJ, 247, L67

Kwok, S., Volk, K. M. \& Hrivnak, B. J. 1989, ApJ, 345, L51

Le Bertre, T. 1988, A\&A, 203, 85

Léger, A., \& Puget, J. L. 1984, A\&A, 137, L5

Lewis, R. S. Amari, S. \& Anders, E. 1990, Nature, 348, 293

Little-Marenin, I. R. 1986, ApJ, 307, L15

Little-Marenin I. R., Ramsay, M. E., Stephenson, C. B., Little, S. J., \& Price, S. D. 1987, AJ, 93,663

Lorenz-Martins, S., \& Lefévre, J. 1993, A\&A, 280, 567

Martin, P. G.. \& Rogers, C. 1987, ApJ, 322, 374

McCabe, E. M. 1982, MNRAS, 200,71

Newman, W. I., Haynes, M. P., \& Terzian, Y. 1994, ApJ, 431, 147

Nuth, J. A., Moseley, S. H., Silverberg, R. F., Goebel, J. H., \& Moore, W. H. 1984, ApJ, 290, L4

Olofsson. H. Eriksson, K. Gustafsson, B. \& Carlstrom, U. 1993, ApJS, 87, 267

Onari, S., Tada, K., \& Toshihiro, A. 1991, J. Phys. Soc. Japan, 60, 4392

Papoular, R. 1988, A\&A, 204, 138

Pégourié, B. 1988, A\&A, 194, 335

Roche, P. F., \& Aitken, D. K. 1983, MNRAS, 203,9P

Rowan-Robinson, M., \& Harris, S. 1982, MNRAS, 200, 197

- 1983, MNRAS, 202,767

Skinner, C. J. \& Whitmore, B. 1988a, MNRAS. 231, 169

. 1988b, MNRAS, 235, 603

Stephenson, C. B. 1973, Publ. Warner \& Swasey Obs., 1, 1

Tang, M., \& Anders, E. 1988, Geochim. Cosmochim. Acta, 52, 1235

Tielens, A. G. G. M. \& Allamandola L. J 1987, Interstellar Processes (Dordrecht: Reidel), 397

Treffers, R., \& Cohen, M. 1974, ApJ, 188, 545

Tsuji, T. 1964, Ann. Tokyo Astron. Obs., 9, 1

. 1973, A\&A. 23, 411

yan der Veen. W. E. C. J. \& Habing. H. J. 1988, A\&A, 194, 125

Volk, K., \& Cohen, M. 1989, AJ, 98, 931

Walker, H. J. 1986, in IAU Collog. 87, Hydrogen Deficient Stars and Related Objects, ed. K. Hunger, D. Schönberner, \& N. K. Rao (Dordrecht: Kluwer), 407

Walker, H. J.\& Cohen, M. 1988, AJ, 95, 180

Warner, B. 1967, MNRAS, 137, 119

Whittet, C. C. B., Duley, W. W., \& Martin, P. G. 1990, MNRAS, 244, 427

Willems, F. J. 1987, A\&A, 203, 65

Willems, F. J \& de Jong, T. 1986a, ApJ, 309, L39

Win 1986, A\&A 196,173

Winters, J. M., Dominik, C., \& Sedlmayr, E. 1994, A\&A, 288, 255

Witteborn, F. C., Sandford, S. A., Bregman, J. D., Allamandola, L. J., Cohen, M., Wooden, D. H., \& Graps, A. L. 1989, ApJ, 341, 270

Wooden, D. H., Rank, D. M., Bregman, J. D. Witteborn, F. C., Tielens, A. G. G. M. Cohen M. Pinto P. A. \& Axelrod, T. S. 1993, ApJS, 88377

Zinner, E., Tang, M., \& Anders, E. 1989, Geochim. Cosmochim. Acta, 53, 3273

Zuckerman, B. 1993, A\&A, 276, 367

Zuckerman, B., \& Maddalena, R. G. 1989, A\&A. 223, L20

Note added in proof.-A similar discussion regarding dehydrogenation of carbon analog grains has appeared in print (V. Mennella et al., ApJ, 444, 288 [1995]) in its application to the $2200 \AA$ band in the interstellar medium. 
